\title{
A novel LEMD3 pathogenic variant in a son and mother with osteopoikilosis
}

\author{
Selin Elmaoğulları ${ }^{1}$, Adelet Elçin Yıldız ${ }^{2}$, Selma Demir ${ }^{3}$, Hakan Gürkan ${ }^{3}$, \\ Seyit Ahmet Uçaktürk ${ }^{1}$ \\ Departments of ${ }^{1}$ Pediatric Endocrinology and ${ }^{2}$ Radiology, Ankara Children's Hematology and Oncology Training and \\ Research Hospital, Ankara; ${ }^{3}$ Department of Molecular Genetics, Trakya University Faculty of Medicine, Edirne, Turkey. \\ E-mail: ekerbicerselin@yahoo.com
}

Received: 10th February 2018, Revised: 22nd June 2018, 23rd July 2018, Accepted: 26th July 2018

SUMMARY: Elmaoğulları S, Yıldız AE, Demir S, Gürkan H, Uçaktürk SA. A novel LEMD3 pathogenic variant in a son and mother with osteopoikilosis. Turk J Pediatr 2019; 61: 594-598.

Osteopoikilosis (OPK) is a rare, benign condition characterized by osteosclerotic foci that occur in the epiphyses and metaphyses of long bones, wrists, feet, ankles, pelvis, and scapulae. We report a 16-year-old boy and his mother incidentally found to have sclerotic lesions on X-ray. Both of them were asymptomatic and the bone scan of the boy ruled out osteoblastic metastases. We have shown that the boy and his mother have a previously unknown pathogenic variant of the LEMD3 gene, supporting the diagnosis of osteopoikilosis.

Key words: osteopoikilosis, LEMD3, pathogenic variation.

Osteopoikilosis (OPK) is a rare, benign condition characterized by osteosclerotic foci that occur in the epiphyses and metaphyses of long bones, wrists, feet, ankles, pelvis, and scapulae. Its prevalence is estimated to be $1 / 50,000 .{ }^{1}$ Osteopoikilosis may present either as an isolated skeletal abnormality or as a component of Buschke-Ollendorf syndrome (BOS) with additional multiple subcutaneous nevi/nodules with or without melorheostosis, all of which are associated with heterozygous loss of function mutations in the LEM domaincontaining-3 (LEMD3) gene on chromosome $12 \mathrm{q} 14 .^{2,3}$ Isolated OPK is mostly asymptomatic and found incidentally on radiographs and computed tomography scans except a few cases who present with bone pain. ${ }^{4}$ Mastocytosis, tuberous sclerosis, and most importantly, osteoblastic metastases should be ruled out for differential diagnosis. ${ }^{5}$ Here, we present a son and mother with osteopoikilosis carrying a novel LEMD3 pathogenic variant.

\section{Case Report}

A 16-year-old boy who had a traumatic fracture on the distal end of the fifth metacarpal of the right hand was admitted to a pediatric endocrinology outpatient clinic for evaluation of the punctate sclerotic lesions seen on X-ray. He did not have bone pain, arthralgia or any systemic symptoms until the fracture occurred. His medical history was unremarkable except traumatic fractures of his shoulder and foot in the past due to severe traumas. Physical examination was normal except the splint on his right hand. He weighed $75 \mathrm{~kg}$ (79 ${ }^{\text {th }}$ percentile) and was $174 \mathrm{~cm}$ tall (54 ${ }^{\text {th }}$ percentile). He did not have any skin changes (lentil-sized white or yellow nodules, plaques or disks containing fibrocollagenous tissue), joint dismobility or contractures that would suggest BOS. Levels of serum calcium, phosphorus, alkaline phosphatase and parathormone were within normal range. Skeletal survey revealed bilateral, millimetric, 


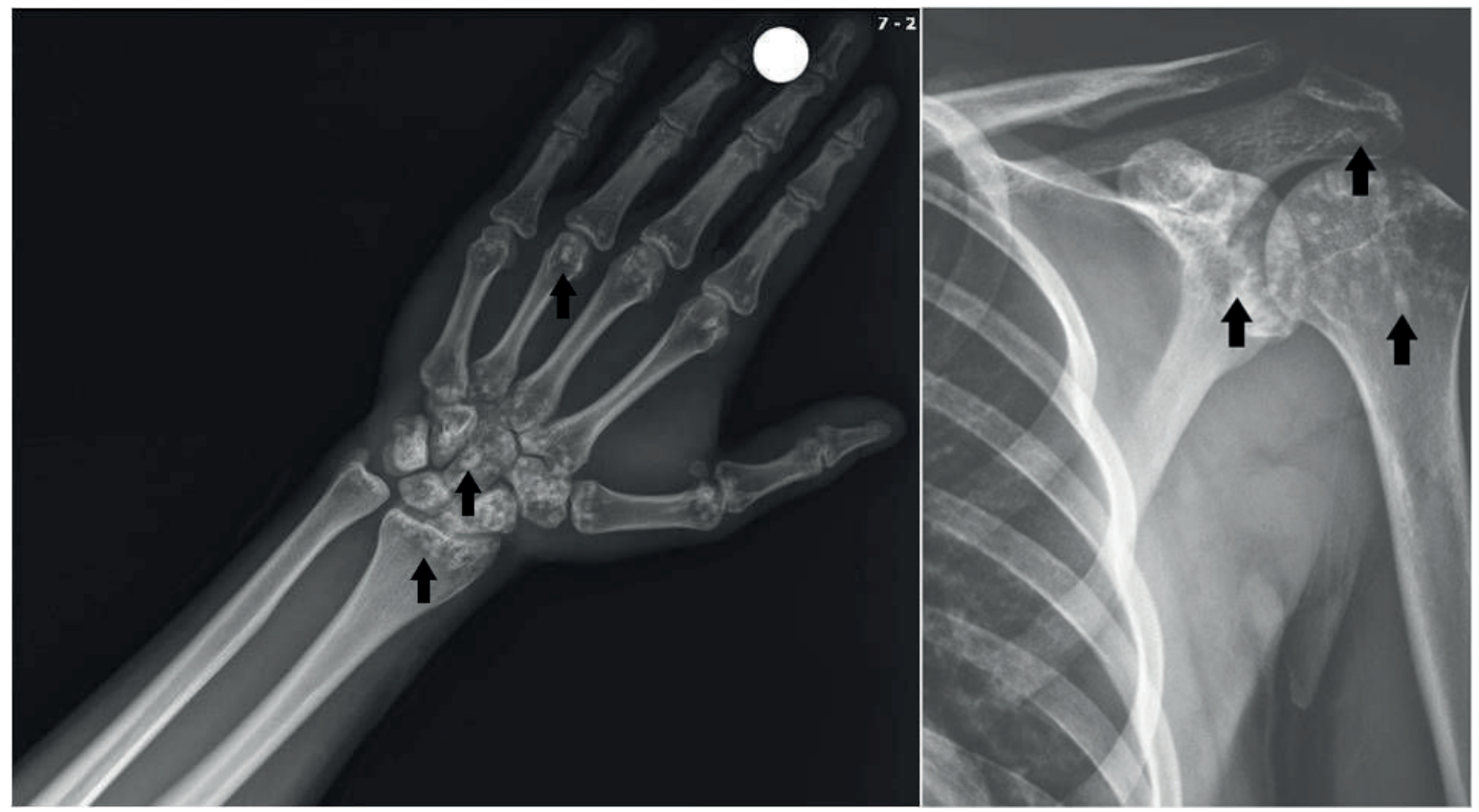

Fig. 1. Images from bone surveys of the proband. (A) Anteroposterior radiograph of the left hand demonstrating multiple sclerotic foci in the distal radius and ulna, carpal, metacarpal and proximal phalangeal bones. (B) Osteosclerotic lesions in both humoral head and scapula. (C) Sclerotic foci of variable size in femur and tibia with periarticular distrubition and predominant epiphsial location.

mostly round-shaped sclerotic lesions on the patient's wrists, elbows, shoulders, hips, knees, ankles, interphalangeal and intertarsal joints (Fig. 1). The whole-body Tc-99m methylene diphosphonate bone scintigraphy performed to rule out osteoblastic bone metastases revealed increased distribution only on the right elbow, wrist and distal metacarpal bones which were attributed to traumatic injury. With the presence of typical radiological findings and exclusion of all the other possible causes, he was diagnosed with OPK.

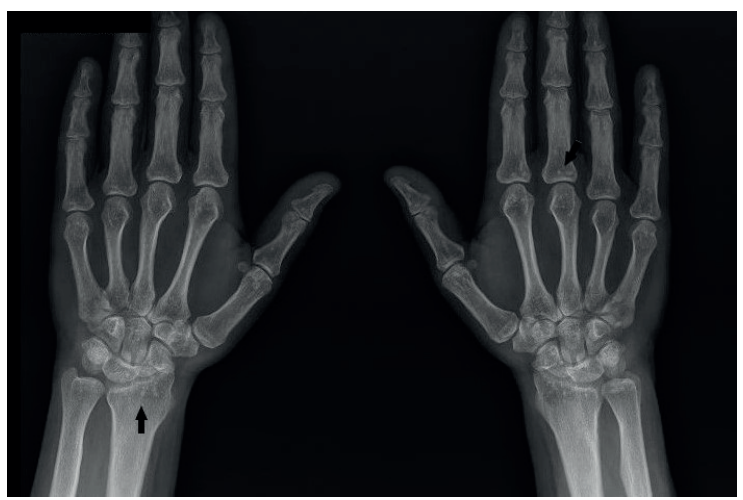

Fig. 2. Bilateral wrist X-rays of the mother showing sclerotic foci in the distal radius and proximal phalangeal bones.
As OPK is a hereditary condition, his parents were also evaluated with wrist graphs, and his mother was found to have the same sclerotic lesions, along with the same unremarkable medical history (Fig. 2).

After obtaining written informed consent, blood samples were taken from the patient and his parents for genetic study. Genomic DNA was isolated from peripheral blood leukocytes. The targeted genomic region was amplified by polymerase chain reaction and sequenced with Nextera Rapid Capture (Illumina) kit on MiSeq System. The variations were verified with Sanger Sequencing method (Fig. 3). A heterozygous c. $2387+2$ dupT variation in exon 10 splice donor region of LEMD3 (transcript NM_014319.4) was detected both in the affected son and his mother. This variant was previously not defined in the Human Genome Mutation Database (http://www.hgmd.cf.ac. uk), ClinVar (https://www.ncbi.nlm.nih.gov/ clinvar/) and Exome Aggregation Consortium (ExAC) database (https://exac.broadinstitute. org/). In silico analysis (Mutation Taster ${ }^{6}$ and $\mathrm{CADD}^{7}$ score:26) predicted that this variation is pathogenic which supported the OPK diagnosis. 

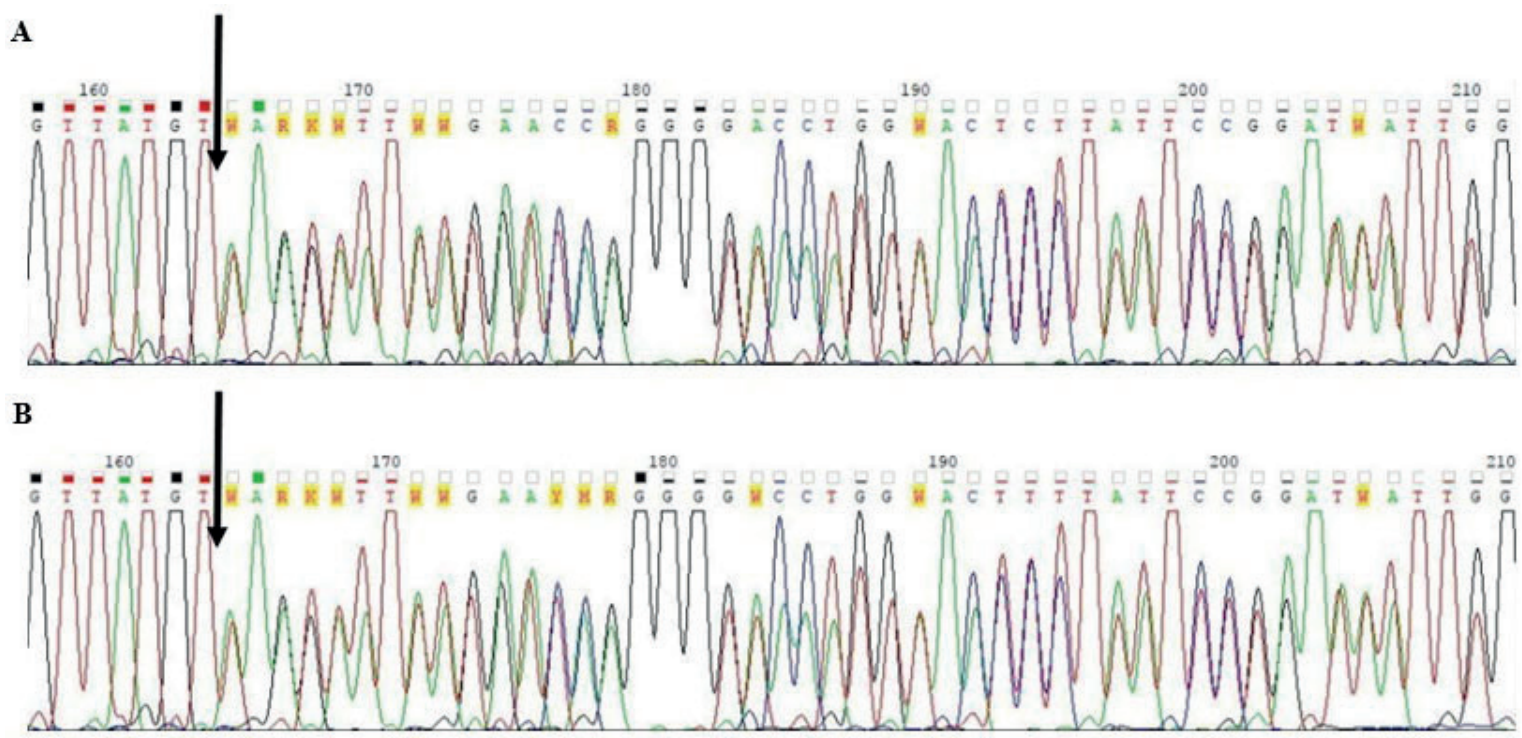

C

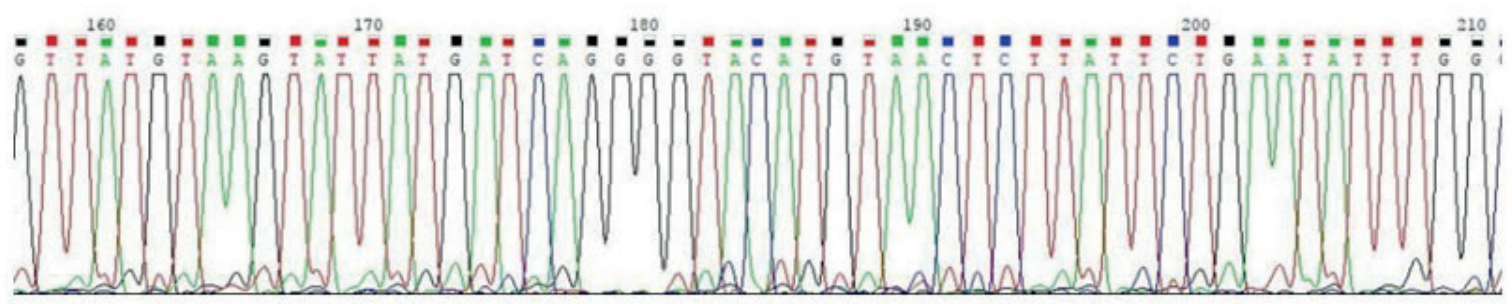

Fig. 3. Sanger sequence analysis of the exon 10 splice region of LEMD3 gene. The arrow indicates the novel heterozygous NM_014319.4:c.2387+2dupT variation in the proband (A) and his mother (B). The father (C) does not have the variation.

\section{Discussion}

Sclerotic lesions of bones require differential diagnosis and work up. The appearance, number, location, and distribution of the lesions give a great deal of information in distinguishing osteoblastic metastases from benign sclerotic lesions.

Osteopoikilosis, is a rare, benign bone disorder. It is usually inherited autosomal dominantly, but sporadic forms have also been reported. ${ }^{8}$ Characteristic lesions of OPK are multiple, punctate, sclerotic, round- or oval-shaped, distributed in a predominantly periarticular fashion within the epiphyseal and metaphyseal regions throughout the axial and appendicular skeleton. ${ }^{1}$ The lesions are usually $1-10 \mathrm{~mm}$ in diameter and symmetric, but unilateral lesions up to $16 \mathrm{~mm}$ have been reported. ${ }^{9}$
Bone scanning is an important tool in patients with a known or suspected primary malignancy and is usually normal in patients with OPK. Radiologic findings of the proband and his mother were characteristic for OPK, however, as previous X-rays to prove that the lesions were stable over time were not available, we performed a bone scan to rule out osteosarcomatosis or osteoblastic metastases. The minimum time for a fracture to heal on bone scan was reported to be 5 months, and in this case the localized increased uptake in the right arm was attributed to the fracture. ${ }^{10}$ Additionally in some OPK cases bone scans can be abnormal reflecting active osseous remodelling. ${ }^{11}$ As the patient had no systemic symptoms, sarcoidosis, mastocytosis and tuberous sclerosis were excluded. ${ }^{1}$ Pachydermoperiostosis, characterized by digital clubbing, pachydermia and subperiosteal new bone formation; and enostosis, characterized by homogeneously dense, sclerotic foci in the 
cancellous bone with distinctive radiating bony streaks creating a brush-like border, are other diseases that we considered in the differential diagnosis. ${ }^{12,13}$

OPK is usually asymptomatic but in $15-20 \%$ of the cases, mild articular pain and joint effusions may be seen. ${ }^{14}$ It can be isolated or in association with other abnormalities of skin, bone, rheumatic diseases, organ anomalies, and endocrine dysfunctions. But these are usually single case reports and most of them seem to be coincidental rather than having causal relationships except BOS, and melorheostosis. ${ }^{1}$ BOS manifest by subcutaneous nevi or nodules in addition to OPK. While some family members may have only skin or bone findings, some family members may have both. Melorheostosis is characterized by asymmetric hyperostosis of the cortex of tubular bones resembling dripping candle wax. It is predominantly a sporadic disorder and may either be isolated or accompany OPK or BOS.

LEMD3 is shown to be the causal gene for OPK and BOS. LEMD3 encodes an inner nuclear membrane protein that antagonize bone morphogenetic protein (BMP) and transforming growth factor (TGF $\beta$ ) signaling pathways. Heterozygous loss of function mutations in LEMD3 result in altered bone formation in OPK through increased BMP, and skin lesions of BOS through increased TGF $\beta .^{2}$ We have found a novel pathogenic LEMD3 variant in our cases, which may be associated with OPK, BOS or melorheostosis. The proband had isolated OPK, however his mother was not screened for all bones so we could not rule out melorheostosis in her. Sclerotic lesions may develop during childhood and persist throughout life. ${ }^{15}$ We plan longitudinal followup to document progression in these cases.

In conclusion, osteopoikilosis is a benign bone disorder characterized by multiple, round- or oval-shaped sclerotic lesions on periarticular regions caused by heterozygous pathogenic LEMD3 mutations. BOS and melorheostosis are associated disorders and osteoblastic metastases is the major diagnosis that should be ruled out.

\section{REFERENCES}

1. Di Primio G. Benign spotted bones: a diagnostic dilemma. CMAJ 2011; 183: 456-459.

2. Hellemans J, Preobrazhenska O, Willaert A, et al. Loss-of-function mutations in LEMD3 result in osteopoikilosis, Buschke-Ollendorff syndrome and melorheostosis. Nat Genet 2004; 36: 1213-1218.

3. Zhang Y, Castori M, Ferranti G, Paradisi M, Wordsworth BP. Novel and recurrent germline LEMD3 mutations causing Buschke-Ollendorff syndrome and osteopoikilosis but not isolated melorheostosis. Clin Genet 2009; 75: 556-561.

4. Mahbouba J, Mondher G, Amira M, Walid M, Naceur B. Osteopoikilosis: A rare cause of bone pain. Caspian J Intern Med 2015; 6: 177-179.

5. Wadhwa SS, Mansberg R. Abnormal bone scan in osteopoikilosis. Clin Nucl Med 1999; 24: 71-72.

6. Schwarz JM, Cooper DN, Schuelke M, Seelow D. MutationTaster2: mutation prediction for the deepsequencing age. Nat Methods 2014; 11: 361-362.

7. Kircher M, Witten DM, Jain P, O'Roak BJ, Cooper GM, Shendure J. A general framework for estimating the relative pathogenicity of human genetic variants. Nat Genet 2014; 46: 310-315.

8. Negi RS, Manchanda KL, Sanga S, Chand S, Goswami G. Osteopoikilosis - Spotted bone disease. Med J Armed Forces India 2013; 69: 196-198.

9. Benli IT, Akalin S, Boysan E, Mumcu EF, Kis M, Turkoglu D. Epidemiological, clinical and radiological aspects of osteopoikilosis. J Bone Joint Surg Br 1992; 74: 504-506.

10. Matin P. The appearance of bone scans following fractures, including immediate and long-term studies. Nucl Med 1979; 20: 1227-1231.

11. Mungovan JA, Tung GA, Lambiase RE, Noto RB, Davis RP. Tc-99m MDP uptake in osteopoikilosis. Clin Nucl Med 1994; 19: 6-8.

12. Alessandrella A, Della CR, Alessio M, Puento PJ, Striscioglio P, Melis D. A novel homozygous mutation in the SLCO2A1 gene causing pachydermoperiostosis: Efficacy of hydroxychloroquine treatment. Am J Med Genet A 2018; 176: 1253-1257.

13. Greenspan A, Stadalnik RC. Bone island: scintigraphic findings and their clinical application. Can Assoc Radiol J 1995; 46: 368-379. 
14. Woyciechowsky TG, Monticielo MR, Keiserman B, Monticielo OA. Osteopoikilosis: what does the rheumatologist must know about it? Clin Rheumatol 2012; 31: 745-748.
15. Morrison JG, Jones EW, MacDonald DM. Juvenile elastoma and osteopoikilosis (the Buschke Ollendorff syndrome). British J Dermatol 1977; 97: 417-422. 